\title{
Sistema de información web para la toma de decisiones en la evaluación de la calidad de productos agroindustriales
}

\section{Web information system for decision making in the quality evaluation for agro industry products}

\author{
ZAPATA-GARAY, Nitgard $\dagger^{*}$, OYARVIDE-MARTINEZ, Oscar Daniel, GARCIA-ALDAPE, \\ Horacio y PIEDAD-RUBIO, Ana María
}

Tecnológico Nacional de México Campus Instituto Tecnológico de Ciudad Valles.

ID $1^{\mathrm{er}}$ Autor: Nitgard, Zapata-Garay / ORC ID: 0000-0002-4060-1826, CVU CONACYT ID: 360443

ID $1^{\mathrm{er}}$ Coautor: Oscar Daniel, Oyarvide-Martínez / ORC ID: 0000-0002-9085-8419, CVU CONACYT ID: 949412

ID $2^{\text {do }}$ Coautor: Horacio, Garcia-Aldape / ORC ID: 0000-0002-0920-9420

ID $3^{\text {er }}$ Coautor: Ana María, Piedad-Rubio / ORC ID: 0000-0003-1258-0383, CVU CONACYT ID: 732279

DOI: $10.35429 / J A D .2019 .7 .3 .26 .31$

Recibido 19 de Enero, 2019; Aceptado 29 de Marzo, 2019

\begin{abstract}
Resumen
La industria piloncillera en la Huasteca Potosina, México representa un mercado en crecimiento a nivel nacional y global. México realiza exportaciones a EUA, Europa y Japón con tasas muy por debajo de países como India, Colombia o Brasil. La poca o escasa tecnificación de este sector en la región se considera un factor de retraso en el desarrollo agroindustrial. El Instituto Tecnológico de Ciudad Valles actualmente realiza estudios para la evaluación de dos de las características de calidad del piloncillo: color y textura; explotando las ciencias computacionales a través del procesamiento de imágenes. El presente trabajo define el desarrollo de un sistema de información mediante la metodología iWeb y técnicas de minería de datos para proveer la información que caracterice al piloncillo como producto de calidad. Con la sistematización de procesos para el control de calidad es posible brindar información directa que apoye a la toma de decisiones y favorezca el crecimiento de la industria hacia mercados globales. Incorporando características de potabilidad de la información, capacidad de tener datos confiables sobre la evaluación del producto y la representación estadística de defectos; convirtiéndose en una herramienta de apoyo para la mejora de la industria piloncillera.
\end{abstract}

Agroindustria, Calidad, Toma de decisiones

\begin{abstract}
The raw cane sugar industry in Huasteca Potosina, Mexico represents a growing market nationwide and globally. Mexico exports to the US, Europe and Japan with below rate such as a country like India, Colombia or Brazil. The little technification of this sector in the region is considered as a factor of the delays in the agro industry development. The Technological Institute of Ciudad Valles currently conducts researches for the evaluation of two of the quality characteristics of the raw cane sugar: color and texture; exploiting the computer sciences through image processing. This project defines the development of an information system through the iWeb methodology and data mining techniques to provide the information that characterizes the raw cane sugar as a quality product. With the systematization of processes for quality control, it is possible to provide direct information that supports decision-making and favors the growth of the industry towards global markets. Incorporating characteristics of information portability, ability to have reliable data on product evaluation and statistical representation of defects; becoming a support tool for the improvement of the raw cane sugar industry.
\end{abstract}

Agro industry, Quality, Decision making

Citación: ZAPATA-GARAY, Nitgard, OYARVIDE-MARTINEZ, Oscar Daniel, GARCIA-ALDAPE, Horacio y PIEDADRUBIO, Ana María. Sistema de información web para la toma de decisiones en la evaluación de la calidad de productos agroindustriales. Revista de Arquitectura y Diseño. 2019. 3-7: 26-31.

\footnotetext{
*Correspondencia al Autor (Correo Electrónico: nitgard.zapata@tecvalles.mx)

$\dagger$ Investigador contribuyendo como primer autor.
} 


\section{Introducción}

Para la elaboración de productos con altos estándares de calidad, es necesario desarrollar nuevas tecnologías que permitan mejorar la producción y al mismo tiempo que aseguren la calidad y la homogeneidad de un producto. El control de calidad surge como una necesidad global en la industria para desarrollar productos que cumplan altos estándares de precisión y desempeño. Algunas empresas del sector industrial involucran complejos procesos de calidad que demandan gran cantidad de recursos humanos y económicos; estos representan un costo que las empresas tienen que asumir y por lo tanto desempeñan un papel importante en el precio final de un producto (Peña-Cañas, 2007) (Pérez-Fernández, 2010).

En el campo del control de calidad dentro de la ingeniería, se están aplicando diversas técnicas de visión artificial, con el fin de automatizar y mejorar los procesos de manufactura (Aguilar-Torres \& Cornelio, 2008) (Armesto, Tornero, Herraez, \& Asensio, 2011) (Mital, 1998). En los últimos años, se ha observado un desarrollo en sectores como la industria de autopartes y la industria de alimentos, en las cuales se logró obtener dispositivos que superan en velocidad $\mathrm{y}$ precisión la mano de obra humana (Fernandez, Sariňana, \& Swenson, 2009). Con la automatización no solo se puede mejorar el proceso, sino que también se recolectan y almacenan datos en tiempo real, los cuales son de gran beneficio para detectar fallas en la producción.

Debido a su capacidad de sistematizar procesos de control de calidad, los sistemas en la nube permiten otorgar información en tiempo real, la cual apoya en la verificación de productos que cumplan con estándares nacionales e internacionales. Es por ello que se diseñó una aplicación Web que integre técnicas de minería de datos para el seguimiento de las líneas o lotes de producción de productos agroindustriales, otorgando a los usuarios (productores) información continua sobre las características de sus productos en determinados tiempos y espacios, que les serán útiles para las tomas de decisiones.
De esta manera su podrá comprobar la hipótesis de que "El desarrollo de un sistema de información permite identificar las características de calidad de productos agroindustriales de manera rápida para la toma de decisiones".

\section{Descripción del Método}

En el presente proyecto se enfoca en el desarrollo de un sistema de información que mediante técnicas de minería de datos da muestra de la evaluación de la calidad de los productos agroindustriales que permita una mejor toma de decisiones. Con la finalidad de contribuir a la tecnificación de los procesos agroindustriales, particularmente en la producción del piloncillo con miras a la exportación, se propone un sistema de información que se desarrolla con base en la metodología iWeb, mediante cinco etapas fundamentales.

\section{Ventajas de sistemas Web.}

Son aplicaciones que los usuarios pueden utilizar accediendo a un servidor web a través de Internet o una intranet mediante un navegador. Es decir, es una aplicación software que se codifica en un lenguaje soportado por los navegadores Web como HTML, entre otros; en la que se confía la ejecución al navegador. (Ramos Martín \& Ramos Martín, 2014).

El diseño y desarrollo de aplicaciones Web consiste en evaluar e implementar las alternativas de solución detallada según las necesidades, objetivos o ideas, en internet, utilizando las tecnologías más idóneas según el proyecto a ejecutar. Las aplicaciones Web son desarrolladas de acuerdo a las necesidades, estas pueden ser de acceso público como tiendas virtuales, diarios digitales, portales de Internet, etc., o de acceso restringido como son las intranets para mejorar las gestiones internas de una empresa como, gestión de proyectos y tareas, control de presencia, gestores documentales, entre otros, o el uso de redes externas para aumentar y mejorar el servicio con los distribuidores, clientes, proveedores, comerciales y colaboradores externos (Contreras Castañeda, 2016). 
Debido a su capacidad de sistematizar procesos de control de calidad, los sistemas en la nube permiten otorgar información para la toma de decisiones en tiempo real, lo cual genera productos que cumplen con estándares internacionales.

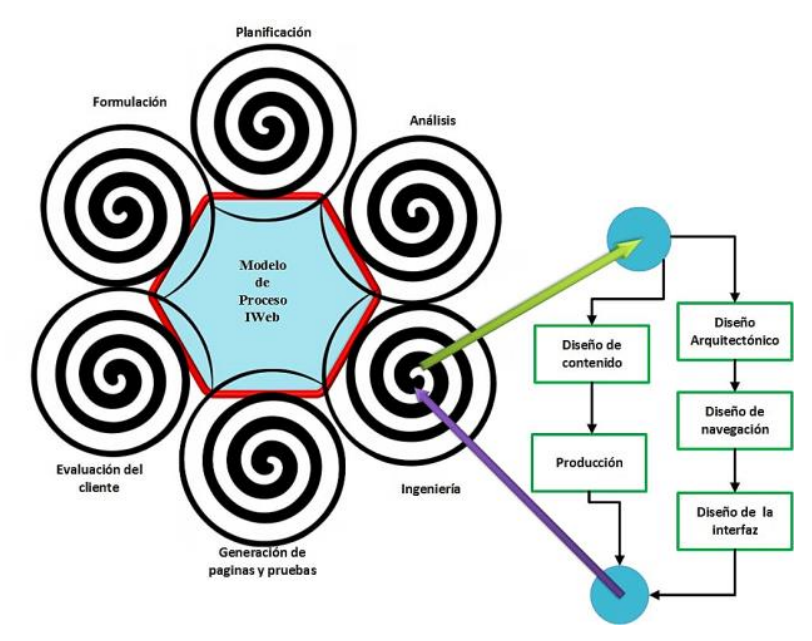

Figura 1 Metodología iWeb

Etapa 1: Diagnostico (Interfaz externa, Arquitectura, Minería de Datos)

Con base en las necesidades técnicas del presente proyecto se determina utilizar una arquitectura de tres capas para el desarrollo de la tecnología web la cual integra una infraestructura tecnológica que puede ser soportada por ambientes Linux o Windows, con lo cual se logra tener una solución multiplataforma.

En la primera capa se contempla la integración del servidor web apache junto con sus módulos base y sus módulos adiciones como lo son el módulo PHP y PDO. Se requiere la configuración de un servicio FTP y SSH para la conexión y carga de archivos del proyecto.

La segunda capa requiere un servidor de base de datos bajo ambientes Linux o Windows que integren el gestor de base de datos MySQL y de igual manera se deberá habilitar el servicio SSH para su conexión en caso de modificación de la configuración.

La tercera capa es la representada por los clientes y/o usuarios que a través de un navegador web hacen uso del sistema de información desarrollado. Este deberá soportar los navegadores más comunes como son:
Google Chrome, Mozilla Firefox, Microsoft Edge, entre otros. Brindando así una herramienta disponible para cualquier equipo de cómputo y/o dispositivo móvil que requiera hacer uso del sistema.

Funcionalmente el sistema permite cumplir aspectos organizacionales $\mathrm{y} / \mathrm{o}$ administrativos planteados en los objetivos del presente proyecto, mediante una estructura flexible y jerárquica que permita tener un control y seguimiento segmentado de los organismos participantes considerados en el proyecto. Las imágenes se obtendrán mediante una interfaz externa al sistema, generando solo el registro de datos para posteriormente aplicarles técnicas de minería de datos para su procesamiento e interpretación en gráficas y/o reportes útiles para la toma de decisiones de los puestos estratégicos en las organizaciones.

\section{Etapa 2: Propuesta.}

Para el cumplimiento de los requisitos funcionales es necesario proponer una interfaz gráfica de usuario amigable y entendible de forma que de una manera intuitiva se pueda hacer uso del sistema de información de manera eficiente y cumpliendo características de responsividad, accesibilidad y disponibilidad que garanticen un correcto funcionamiento a toda hora y en todo momento.

Es necesario tener configurado un entorno de desarrollo para aplicaciones web que integre el manejo de códigos en: HTML5, CSS3, JQuery, Bootstrap, PHP, entre otros. Para la carga y/o actualización del ambiente web es necesario contar con un cliente FTP que tenga acceso a la arquitectura de servidores propuesta.

\section{Etapa 3: Desarrollo.}

En la fase de desarrollo se realiza la codificación del sistema de información cumpliendo con los requisitos funcionales. En relación al aseguramiento de calidad y seguridad del sistema se realizan una serie de pruebas que nos garanticen el correcto funcionamiento y consistencia de la información registrada en los procesos planteados en los casos de uso anterior. Por lo cual se genera una serie de matrices de prueba que serán utilizadas para verificar la calidad y seguridad de la aplicación web desarrollada. 


\section{Etapa 4: Evaluación.}

En esta etapa al terminar de construir los módulos de la aplicación web, complementándolo con los contenidos diseñados y creados en la fase de desarrollo, ya que a partir de esa etapa se generarán páginas web en HTML y PHP enfocadas al diseño, para posteriormente realizar la codificación con JavaScript y Ajax que contiene la parte importante, específica y funcional de la App Web. Por lo anterior se determina la realización de pruebas de:

Contenido para identificar errores sintácticos, semánticos y errores en la organización o estructura del contenido final.

\section{Interfaz para identificar problemas en:}

- Vínculos: Con el objetivo que los apartados se cumplan, y así constatar que el contenido mostrado sea el correcto.

- Formularios: Se corroborará que las etiquetas encargadas de identificar los campos dentro del formulario fueran las correctas, que el envío de toda la información sea recibida por el servidor, sin perder datos en la transmisión cliente servidor.

- HTML dinámico: Se verificará que el despliegue de cada página web dinámica sea el correcto.

\section{Usabilidad para revisar las características de:}

- Interactividad: La sección de menú, salir y los botones de acción sean relativamente fáciles de entender y manejar para el usuario, ya que el diseño será creado para reducir la desorientación o confusión al momento de estar interactuando.

- Plantilla: La parte funcional de la aplicación se mostrará rápidamente a través del menú, dejando de entrada el dashboard con información puntual y estratégica de los diagnósticos sin la necesidad de entrar a algún apartado específico.
- Estética: Se revisará que los contenidos (tablas, graficas, texto y los cuadros) adopten un tamaño adecuado, además de que el texto estuviera grande y con un color que permitiera identificar con facilidad cada una de las palabras.

- Características de despliegue: Aprovechar de manera óptima el tamaño y resolución de la pantalla, estableciendo los espacios adecuados para las partes que conforman la aplicación, y resulte cómoda su navegación.

Compatibilidad para llevar a cabo esta prueba se tendrá que probar la aplicación en diferentes navegadores como por ejemplo Chrome, Firefox, Microsoft Edge, Internet Explorer, entre otros; a su vez en distintas computadoras ya sea de escritorio o laptops con diversos sistemas operativos. Se contempla una evaluación en dispositivos móviles como teléfonos inteligentes y tabletas electrónicas, para verificar su adaptabilidad y responsividad.

Navegación esta fase deberá comenzar en conjunto con la prueba de interfaz, esto para tener la certeza de que cada sección de la aplicación web realice sus funciones correctamente, como lo es:

- Vínculos de navegación: Se deberá confirmar que los vínculos colocados en cada módulo cumplan con la función al darle clic.

- Redirecciones: Aquí se validan las llamadas URL, que para este caso se utiliza el tipo de ruta relativa al documento, recomendada para manejar vínculos locales, la cual no permiten conocer realmente en donde se encuentra la codificación de cada apartado en el momento de la navegación.

Componentes también llamada prueba de función, se llevará a cabo para determinar errores en las funciones de la webapp.

Seguridad se revisará que ninguna persona ajena al desarrollado de la App Web tenga los permisos para cambiar la información contenida en los módulos.

Etapa 5: Integración. 
El proceso de integración es una actividad en la cual se busca la vinculación con productores de la región con quienes se trabaja desde el proceso mismo de la definición de esta necesidad. Dicha actividad plantea la instalación de la infraestructura propuesta que va desde la obtención de los datos hasta el despliegue de reportes y gráficas para el apoyo en la toma de decisiones.

\section{Infraestructura propuesta}

La arquitectura de las aplicaciones Web suele presentar un esquema de tres niveles. El primer nivel consiste en la capa de presentación que incluye no sólo el navegador, sino también el servidor web que es el responsable de dar a los datos un formato adecuado. El segundo nivel está referido habitualmente a algún tipo de programa o script. Finalmente, el tercer nivel proporciona al segundo los datos necesarios para su ejecución (Berenguel Gómez, 2015). Una aplicación Web típica recogerá datos del usuario (primer nivel), los enviará al servidor, que ejecutará un programa (segundo y tercer nivel) y cuyo resultado será formateado y presentado al usuario en el navegador (primer nivel otra vez), (ver Figura 2).

Primer nivel

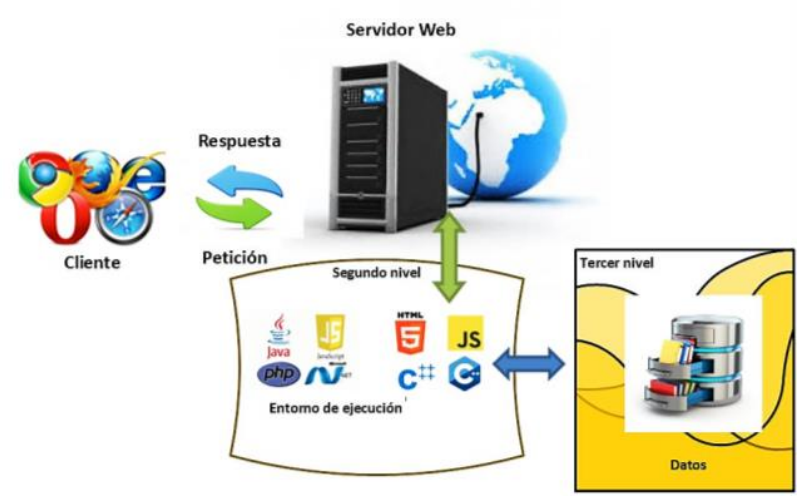

Figura 2 Arquitectura web de tres niveles

\section{Minería de Datos}

Las técnicas de la minería de datos provienen de la Inteligencia artificial y de la estadística. Dichas técnicas, no son más que algoritmos, más o menos sofisticados que se aplican sobre un conjunto de datos para obtener unos resultados.
El hecho es, que en la práctica la totalidad de los modelos y algoritmos de uso general en minería de datos —Redes neuronales, Árbol de decisión, regresión lineal y clasificación, modelos logísticos, análisis de componentes principales, etc. - gozan de una tradición relativamente larga en otros campos.

\section{Conclusiones}

La necesidad del sector agroindustrial de la región por expandirse, demanda la creación de herramientas de monitoreo de los procesos, para su análisis y posterior toma de decisiones. Es fundamental que estas herramientas estén disponibles 24/7; es por ello que el presente trabajo se enfoca en establecer la plataforma de comunicación de las características que se desean estudiar para presentárselas al usuario final de una manera clara y precisa para tomar decisiones de manera eficiente.

Esta plataforma está diseñada para cumplir con la característica de que pueda aplicarse a un amplio sector de la agroindustria $\mathrm{y}$ no a un solo producto o proceso. Es un mecanismo mediante el cual se podrá orientar a los productores sobre la proyección y control de calidad de sus productos mediante gráficas y resultados estadísticos generados mediante técnicas de minería de datos.

\section{Recomendaciones}

Los investigadores interesados en continuar con mejoras a la propuesta planteada podrían concentrarse en la creación de un módulo de Diseño Experimental que pueda identificar cuáles son las materias primas idóneas para la elaboración del producto agroindustrial determinado.

\section{Referencias}

Aguilar-Torres, M. A., \& Cornelio, Y. (2008). A real time artificial vision implementation for quality inspection of industrial products. InElectronics, Robotics and Automotive Mechanics Conference. IEEE, 277-282.

Armesto, L., Tornero, J., Herraez, A., \& Asensio, J. (2011). Inspection system based on artificial vision for paint defects detection on cars bodies. InRobotics and Automation (ICRA). IEEE International Conference, 1-4. 
Berenguel Gómez, J. L. (2015). Desarrollo de aplicaciones web en el entorno servidor. España: Paraninfo.

Contreras Castañeda, M. Á. (2016). Desarrollo de aplicaciones Web multiplataforma. España: Ministerio de Educación.

Fernandez, Y. E., Sariňana, A., \& Swenson, R. L. (2009). Development of a prototype for classification of potato mini-tubers based on artificial vision. In Electrical Engineering, Computing Science and Automatic Control. (págs. 1-6). IEEE.

Huamán-Huamán Wilson, P., \& Olavarría-Paz, J. B. (2018). Diseño de un sistema de información para la mejora Del Proceso de logística y control de almacén utilizando la metodología RUP para la Municipalidad distrital de Sóndor, Provincia de Huancabamba, Departamento de Piur. Recuperado el 2019, de http://repositorio.unprg.edu.pe/bitstream/handle /UNPRG/4015/BC-TES-TMP-

2834.pdf? sequence $=1$ \&isAllowed $=\mathrm{y}$

Lujan Ventura, A. J., \& Rosario Loyola, C. A. (2018). SISTEMA DE INFORMACIÓN WEB PARA AGILIZAR LOS PROCESOS EN EL ÁREA AGRÍCOLA DE LA EMPRESA RIO GRANDE SAC DE OLMOS. Obtenido de http://dspace.unitru.edu.pe/bitstream/handle/U NITRU/11188/Rosario\%20Loyola\%2c\%20Ces ar\%3b\%20Lujan\%20Ventura\%2c\%20Alberto.p $\mathrm{df}$ ? sequence $=1 \&$ is Allowed $=\mathrm{y}$

Mital, A. G. (1998). A comparison between manual and hybrid methods in parts inspection. Peña-Cañas, L. A. (2007). Estudio para la reducción de los costos de producción mediante la automatización de los finales de linea de la planta dressing en la empresa Unilever andina Colombia ltda. Santiago de Cali.

Pérez-Fernández, J. A. (2010). Gestión por procesos. ESIC.

Ramos Martín, A., \& Ramos Martín, M. J. (2014). Aplicaciones Web. España: Paraninfo.
Schomwandt, D., Lucioni, N., Calabrese, L., Dolzan, A., Worcel, L., Baigorri, N., . . . Carpena, A. (2018). La infraestructura de Datos Espaciales como herramienta de integración frente a la. (pág. 120). San Juan, Argentina: IDERA. Obtenido de http://www.observatorio.gov.ar/media/k2/attach ments/Libro_Ponencias_XIII_IDERA_SanJuan _OK.pdf

Solano, A., \& Camilo, A. (2019). Plan de mejoramiento para la comercialización de productos agroindustriales en la empresa Bayen Ltda. Obtenido de https://hdl.handle.net/10983/23344

Zartha, J. W., Álvarez, V. T., Oviedo, J. C., \& Orozco, G. L. (2019). Propuesta de Árbol Temático y Tecnológico sobre la Ingeniería Agroindustrial como Herramienta para un Estudio de Prospectiva. Obtenido de https://scielo.conicyt.cl/scielo.php?script=sci_ar ttext\&pid=S0718-50062019000400013 